but if the bit of dead chorion is left behind fresh poison will be produced, and the symptoms will return. To cure the patient it is necessary that the dead tissue should be removed, and scraping out the uterus is one way of doing this. It is not always the best way, for if the piece of chorion is large it can be better removed by grasping it with forceps constructed to hold. (The so-called "ovum forceps" is useless; it will not hold anything.) When the big mass has been removed then the uterus can be scraped to detach smaller bits and the cleansing process completed by washing out the aterus with a germicide solution. If the case is only one of sapraemia this will cure the patient.

Scraping the uterus is not a remedy for pain. The writer who thinks endometritis so common in virgins has found that this is so, for he says: "For really bad cases in which there is prolonged haemorrhage which repeated curettings have failed to cure, there is a general agreement that vaginal hysterectomy is justifiable. For my part, $I$ think the operation ought to be extended to some cases in which haemorrhage is not a very important feature. There are a good many women whose lives are rendered miserable by chronic metritis at a time when they ought to be most active. If in a case of this sort the uterus is found to be much enlarged and very hard, and everything points to its being functionally useless, and no improvement is brought about by repeated curettirg (italics mine), it ought to be removed." My experience agrees with that of this author, that scraping does not relieve pain. But I hold the opinion, which he does not express, that repeated scrapings, after one scraping has failed to relieve pain, is bad practice, and deserves to be called an "abuse of the curette." The use of the scraper is to detach something that is causing trouble, not to relieve pain.

\section{$\nVdash_{\mathfrak{E m} a r k s}$}

on

\section{PREGNANCY COMPLICATED BY FIBROMYOMATA OF THE U'TERUS, \\ A RECORD OF TEN CASES TREATED BY LAPAROTOMY*}

\section{By F. W. N. HAULTAIN, M.D., F.R.C.P.,} GXNAECOLOGIST, DEACONESS HOSPITAL, EDINBURGH.

By a happy provision of Nature uterine fibromyomata of sufficient import to give rise to symptoms per se are but seldom associated with pregnancy, for there can be no reasonable doubt that their coincidence forms a most serious menace to life.

Curiously, there is a small faction of writers on the subject who look upon fibroids as due to sterility and not as its cause, and who must put, therefore, a large premium on marriage. Statistics and practical experience do not, to my mind, support this contention. From the records of the Edinburgh Royal Infirmary it appears that in 100 cases of fibromyomata 37 were single and 63 married; 32 of the married were childless- 50 per cent.-a very striking proportion when compared with the generallratio of sterility-namely, 10 per cent.

My own experience entirely coinsides with these figures. Out of 290 cases of uterine fibromyomata upon which I thought it necessary to perform hysterectomy before the climacteric, 111 were unmarried and 179 married; of the latter 82 had no children and 97 had among them 222 children. Thirty-one of these had but 1 child each, and the largest family of any was 6. The most outstanding feature, however, was the relative sterility, the youngest child averaging 7 years in those who were not widows; the age of the mothers averaged 41 . It would thus appear that women, the victirns of small fibroids, frequently become pregnant; this, however, acts materially on the tumour in the majority of cases by permanently increasing its growth, so that it tends to prevent future pregnancy, with a resulting relative sterility.

\footnotetext{
* Read before the Edinburgh Obstetrical Society, July, 1908
}

From these statistics one may legitimately form the conclusions-

1. That fibroids occur equally in married and unmarried.

2. That they predispose to sterility.

3. That pregnancy, should it occur, excites the growth of the tumour, and thus predisposes to subsequent sterility:

As is well known, fibroids of pathological import, from their site or size, are occasionally associated with pregnancy, but the complication is much less frequent than is supposed. On consulting the case-books of the Edinburgh Maternity Hospital, and those of Ward 35 in the Royal Infirmary, their rarity must be conceded, as in the former I find but seven cases in ten years out of over 4,000 cases, while during the same period in the latter's records only five cases are mentioned.

From these statistics it may be contended that the coincidence is so trivial as to in the former give rise to no difficulty, and in the latter to warrant no advice being sought. This, from experience, I cannot endorse, for during my tenure of office in the Maternity Hospital I have on but three occasions seen fibromyomata larger than a cocoanut; in one of these cases Caesarean section had to be performed, while another died from eclampsia.

The smaller fibroids, of which I have seen several, were of the innocuous subserous variety, comparable to warts, which gave rise to no symptoms, before, during, or immediately after pregnancy.

The mere presence of a fibromyoma in the wall of a pregnant uterus cannot be considered a serious complication, but when it is large enough or placed' in such a position as to give rise to symptoms of sufficient gravity to warrant the woman seeking advice in the unimpregnated state, there is no doubt in my mind that the coincidence of pregnancy (which is fortunately rare) is undesirable, as it forms a very grave complication. One must clearly distinguish, then, between the occurrence of pregnancy. with innocuous fibroids, and its occurrence with those which in themselves have given rise to signs and symptoms sufficient to attract notice.

The dangers of the latter may be tabulated as_follows:

1. Pressure from their position or size.

2. Degenerations during pregnancy or after labour.

3. Interference with pregnancy and labour.

4. Haemorrhage duri $\mathrm{g}$ or after expulsion of uterine con tents.

5. Secondary post-partum haemorrhage.

6. Readiness to serious septic infection after imperfect expulsion of ovum.

I have had experience of all these complications, but it is my intention to restrict myself to the discussion of 10 cases in which $I$ felt it incumbent to perform laparotomy. They are as follows :

CASE I.

Mrs. R., aged 35, primipara, after three months' amenorrhoea, complained of very severe intermittent pain and sickness, which had been present for over a fortnight. On examination the uterus was found to reach almost as high as the uppilisus and was unequal in consistence. As the result of sleeplessness and want of nourishment, she was becoming very exhausted, and as her temperature rose nightly to between $101^{\circ}$, and $102^{\circ}$; I decided to remove the tumour, which I attempted; as, however, it was so intimately connected with the uterus and the supravaginal hysterectomy.

CASE II.

Mrs. S., aged 35, after six months' amenorrhoea, complained of retention of urine and pain and swelling of lower limbs. On examination the pelvis was found to be filled by a large fibroid, which pushed the uterus up into the abdomen and rendered the cervix difficult to reach high above the pubis. I performed laparotomy, and, having found the pelvic swelling to be an incarcerated subperitoneal fibroid with a comparatively small attachment to the uterus, I removed it easily by transfixing the attachment to the uterus, I removed it easily by transfixing the pedicle and subsequently stitching over the stump with Lembert
sutures. The pregnancy continued to full time and ended in a natural delivery.

CASE III.

Mrs. D., para, iast living child 7 years old, two miscarriages, complained, after six months' amenorrhoea, of a large and rapidly increasing abdominal swelling. For the previous week she had had severe intermittent pains and considerable haemorrhage, and was much exhausted. A large tender, multinodular abdominal swelling could be felt. I opened the abdomen and performed panhysterectomy. The uterus and tumour weighed $18 \mathrm{lb}$. The former showed a dilated cervix and much attenuated. L.U.S. labour was well advanced, but expulsion of the ovum impossible. 
CASE IV,

Mrs. L. was admitted into the Maternity Hospital in laboureight and a half menths pregnant. The abdomen was much distended by an irregularly-shaped uterus, and the pelvis filled by tended by an irregularly-shaped uterus, and the pelvis filed by the examining finger. I performed Caesarean section and subsequently panhysterectomy. The enucleation of the pelvic tumour, which proved to be a cervical fibroia, was associated with very severe bleeding. The woman died the following day from exhaustion, probably due to loss of blood.

CASE v.

Mrs. J., two-para, aged 40, first child 10 years old, came under my care suffering from severe menorrhagia, due to fibromyoma.' I reoommended hysterectomy, but as the patient was bent if possible on having another child I consented to try and abate the bleeding by curetting, warning her that in the first instance I thought her chances of pregnancy were very remote, and if such did take place that it would be fraught with danger. The curettage was fairly successful as regards the haemorrhage, and she returned to india. - A year later I hear that she had ceased menstruating for eleven months, and had developed a large abdominal swelling, with all the signs of pregnancy. Fetal movements had, however, ceased at the end of the eighth month, and at the time of expected confinemen she had thirty-six hours of intense pain, without any dilatation of the cervix. A second bout of severe pain occurred a month later, and lasted twenty-four hours without any appreciable difference in the condition. She was therefore sent home to be under my care. On arrival, I found an abdominal tumour reaching 5 in. above the umbilicus, and by pelvic examination several hard nodules in the cervix. I therefore performed laparotomy, 362 days after her menstruation had ceased, and found a pregnant uterus with cervical fibroids. The liquor amnii a pregnant uterus with cervical fibuor amnii. was almost entirely absorbed. From my previous experience with cervical fibroids and pregnancy I thought it safer to perform supravaginal hysterectomy, from which she made an uninterrupted recovery.

\section{CAsE VI}

Mrs. B., primipara, after four and a half months' amenorrhoea, complained of abdominal swelling, and great pain and tenderness over the abdomen, with much fever, temperature $102.6^{\circ}$ Examination revealed a large multinodular abdominal swelling of varying consistency and marked tenderness over one nodule. Suspecting necrobiosis and pregnancy, I operated withou delay, and removed supravaginally the pregnant uterus with multiple fibroids, one of which was distinctly necrobiotic. (See specimen.)

I saw this patient with Dr. James Ritchie two months previously, and recommended waiting till the child was viable, and if necessary performing Caesarean section and hysterectomy.

Case vir.

Mrs. F., aged 33, primipara, after three months' very irregular waginal haemorrhages, complained of rapid enlargement of :abdomen, retention of urine, and much swelling and pain in the lower limbs. A multinodular abdominal tumour of varying consistency was felt reaching to the umbilicus, and at the time filled the true pelvic brim. This I removed by supravaginal filled the true pelvic brim. This
hysterectomy. (See specimen.)

\section{Case viri.}

Mrs. G., aged 32, primipara, after three months' amenorrhoea, suddenly developed feverish symptoms-temperature $102.8^{\circ}$ and with slight inte mittent abdominal pain expelled a small decidual mass from the vagina. The feverish symptoms continued, and the abdominal pain became constant, and remained so for six days. On the seventh day symptoms of acute peritonitis supervened, and on being called in 1 immediately opened the abdomen, which. I found full of pus oozing from a nodule in the uterine wall. I therefore removed the uterus, which was the seat of two suppurating fibroids. Unfortunately, the patient the seat of two suppurating fibroids. Unfortunately, the patient
died within twelve hours after. The pus showed infection by died within twelve hours
the Streptococcus pyogenes.

CASE IX

Mrs. J., primipara, after three months' amenorrhoea, aborted with such severe bleeding as to require intrauterine plugging. Three days later the temperature rose to $101.8^{\circ}$, and she remained in this feverish condition for four weeks. At the same time severe abdominal pain and distension was complained of. On seeing her in cansultation. I decided to open the abdomen, and found a nodulated uterus, surrounded by pus pockets shut off from the general peritoneal cavity by omental and intestinal $=$ dhesions. 1 therefore cleared out the pelvis and drained through the posterior fornix, with a successful result. The aterus showed a suppurating fibroid, the organism infecting being the staphylococcus.

Case X.

Mrs. J., two-para, aborted at the fourth month imperfectly, and the secundines had to be removed with difficulty by scraping. Three days later she commenced to show feverish symptoms, with some offensive discharge. The uterus was much, enlarged, irregular in outline, and tender, and on the introdpction of the finger a sessile and pulpy mass was felt proceeding into the uterus. As her condition became very grave and signs of peritonitis present, I performed laparotomy with panbysterectomy and thoroughly irrigated and drained the peritoneal cavity, with a most satisfactory result. The uterus was the seat of numerous fibroid nodules, one of which uterus was the seat of numerous fibroid nodules, one
was sloughing and discharging into the uterine cavity.

From the above series it will be seen that laparotomy was performed four times during pregnancy, twice during labour, three times after expulsion completed, and once after labour had ceased without expulsion. The last is, I think, unique, and it is difficult to understand how uterine rupture was prevented.

During pregnancy, three of the four cases required operation on account of the pain which was induced by the presence of the growths. In one of these commencing circulatory degenerative changes in one of the nodules is evident, which doubtless would have gone on to so-called necrobiosis. This is characterized by swelling oedema and pigmentary change from haematine staining, which gives the tumour the appearance of hepatization, and is doubt less due to a variety of red infarction from disturbance of the venous return. It is frequently met with in tumours associated with pregnancy, but not necessarily, so, as I have twice removed similar conditions where no pregnancy existed. Its main characteristic sign is not merely the pain and rapid growth, but the extreme tenderness of the nodule on pressure. Fever is frequently, though not always, present.

In the second case pain was intermittent, and was probably of the nature of uterine contractions which were unable to expel the contents. These recurred at frequent intervals, rapidly reduced the strength of the patient, and gave rise to severe symptoms, which indicated to me the necessity of immediate removal.

The remaining two cases operated on during pregnancy demanded such treatment from the severe pressure symptoms complained of.

The three cases of infection of the tumour by organisms are of interest. In that which was fatal the streptococcus seems to have got access to the growth before expulsion had commenced, while in the others a staphylococcic infection occurred subsequently. All, however, go to demonstrate the readiness with which fibromyomata may be infected, and if taken in conjunction with two similar fatal cases I have seen unoperated on, form an important series.

The frequency with which these tumours become infected post partum is the gravest feature of their coincidence with pregnancy.

This liability to infection is readily explained when one remembers the great increase of the growths and their great vascularity during pregnancy. After expulsion of the ovum, the involuting uterus, by its strong retraction, seriously interferes with their circulation-so much so, indeed, that necrosis and sloughing may occur withou infection; so they thus form a ready nidus for the growth and development of pathogenic organisms should infection occur.

Case vIIr is of particular interest, as infection occurred spontaneously, and gave rise to pain and fever, which were present before the expulsion of the decidual mass.

\section{Diagnosis.}

The diagnosis of the complication was, in the majority of cases, simple, from the history of amenorrhoea and the detection. of excessive enlargement of the uterus, which was irregular in outline and variable in consistency. The absence of amenorrhoea in one case, however, seriously affected the ease of diagnosis, but the mammary changes and varied consistency of the tumour led me to a correct discernment.

Most difficult, however, is the differentiation from these cases of fibromyomata with exaggerated glandular endo. metritis which are associated with amenorrhoea, of which I have seen two examples, one of which I have shown tonight. I must say on both these occasions I diagnosed a pregnancy which did not exist.

On the whole, however, it may be said that the diagnosis of pregnancy and fibroids is comparatively simple.

Prognosis.

On this question we at once come to debatable ground, and it would be useless to attempt to dwell on the views of different authorities. This divergency of opinion, how ever, seems to be more apparent than real, the crux of the situation not being duly defined-namely, pregnancy and 
fibroids generally, as against pregnancy with fibroids whose size and site give rise to symptoms.

In the latter their coincidence is rare, but undoubtedly grave. In a comparatively large experience I have on only three occasions seen pregnancy occur in individuals who have previously known of the presence of the growths. Two of these are cases cited as requiring laparotomy; the third had a large fundal tumour, and had no difficulty with pregnancy or labour beyond a somewhat severe puerperal bleeding.

In this connexion, the question of marriage in women with known fibroids is one of importance.

Fortunately, from the usually late development of these growths, one is seldom consulted on this question, as the individual is somewhat passé so far as bridal developments are concerned; still, exceptions do occur. Should they do so, I certainly would be no partisan to negative the proposal, mainly from the improbability of pregnancy occurring. I would, however, warn the patient of the seriousness of the off-chance, and leave it for her own decision. In other words, a woman with a known fibromyomata is better single, but, if married, safer sterile. As I have already stated, I have, had 2 deaths in the 10 cases operated on - one from septic peritonitis before the operation was undertaken, and the other from haemorrhage after enucleation of a large cervical fibroid at term. This in itself forms the large mortality of 20 per cent., but when to this is added two fatalities seen unoperated on, the serious nature of the complication is materially increased.

Speaking generally, small innocuous fibroids-which, according to statistics from post.mortem examinations, occur in 20 per cent. of women-are of no moment, but the small residue which in themselves give rise to symptoms must be considered of very anxious import when associated with the gravid state.

\section{Treatment.}

The treatment of cases of pregnancy complicated by fibroids is a subject of the greatest importance, and neces sarily must vary with each individual case. Generally, it may be said to be primarily expectant in every instance, as, according to many observers, tumours of a size and position which at first would seem to make it appear unlikely that pregnancy and labour could be safely termi nated, have been shown to have given rise to no untoward results whatever. Of these, however, I have no such happy personal experience, although in two of the cases previously described I had the possible opportunity of doing so, as the patients were under my care before urgent symptoms supervened.

Management on these lines must only, however, be adopted if it can be shown that by conserving the life of the child the risk to the mother is not seriously increased. This can, in the majority of cases, be answered in the affirmative from the following reasons :

1. The chance of no complications occurring.

2. Should complications arise they can usually be treated successfully.

3. The risk of operative treatment after the viability of the child, though increased, is not sufficient so as to warrant early interference.

4. The dangers during and after expulsion appear to be as great in the early as in the later months of pregnancy.

The lines of active treatment at our disposal are

The induction of abortion.

Myomectomy.

The first of these is to be entirely deprecated, and should never be resorted to. This assertion I make for two cogent reasons-first, the dangers of abortion are very great, as evidenced by the cases cited, and in my experience supplemented by two other fatal cases from septicaemia. Further, should fortunately the woman survive the risks of abortion, it is almost certain she will have to undergo further treatment by myomectomy or hysterectomy, which could have been as safely done with the uterus gravid as after it had been emptied. By the induction of abortion, therefore, the patient has to run the double risk of the abortion and subsequent operation, of which the former is by far the greater.
From a conservative standpoint myomectomy, when practicable, is the ideal treatment, but in the majority of cases it is, unfortunately, unsuitable, from the multiplicity of the growths, their degenerations, and sessile character.

In the treatment of tibroids generally, myomectomy is shown by statistics to be mcre dangerous than hysterectomy. If this be so, it is much increased in the gravid uterus, from the increased vascularity, which must necessarily tend to increased bleeding, not to mention the risks of subsequent abortion. With a solitary growth, however, giving reasonable hope of ready enucleation, myomectomy should be attempted in the majority of cases. More frequently, however, hysterectomy will form the most satisfactory and safe operation.

During pregnancy, the operation is more easily performed than usual, due to the softness of the surrounding tissues and the laxity of the peritoneum, and seems to be associated with little more risk than when undertaken in the non-gravid state, although from increased vascularity haemorrhage may prove troublesome to control, partieularly in the enucleation of intraligamentary and cervical growths.

Conclusions.

The important points which may be gleaned from what has been said may be summed up in the following con. clusions :

1. Fibroids tend towards sterility.

2. The association of pregnancy with fibroids in themselves sufficient to give rise to symptoms, is an extremely anxious complication.

3. That treatment in all cases should be expectant, but on complications arising myomectomy or hysterectomy should be adopted.

In bringing the important subject before the society, $\mathbf{I}$ am well aware I am treading on debatable ground, but its importance has, I feel, warranted me stating my experience as a text for a general review upon it. Many individual cases have been described and specimens shown, bat general discussions on this complication are rare.

My experience roay have been exceptional, and is so when compared with that of Champneys, Spencer, and others, ${ }^{1}$ who have seldom found the coincidence of fibroids and preguancy of anxious moment.

From this experience I may have taken too pessimistic a view of the subject, and this has prompted me to court discussion, as there can be little doubt that the rapid advance of abdominal surgery has entirely changed the treatment of these growths within recent years, and the complication of fibroids and pregnancy though undoubtedly anxious is no longer associated with the huge mortality of twenty years ago.

1 Proceedings Royal Society of Medicine, May, 1908.

HARVARD UNIVERSITY has received from the Pasteur Institute of Paris a replica of the bust of the great discoverer, by M. Paul Dubois. It will be placed in the Medical School.

WE learn from the Australasian Medical Gazette that the question of providing additional accommodation for patients suffering from pulmonary tuberculosis in an advanced stage. has again been made the subject of representation to the Victorian State Government by Dr. Norris, Chairman of the Victorian Board of Heath. The metropolitan councils have considered the question of accommodation in invalid homes, as distinguished from sanatoriums. There are at present about 120 beds available for this purpose, and probably 150 more are requifed. Dr. Norris points out that should the councils determine to provide an institution or institutions for such patients, the Government, under the Health Act, is liable for half the cost. It is therefore, he says, necessary to consider whether it would not be preferable for the Government itself to establish such an institution, and to arrange with the councils concerned for the admission of patients on the basis laid down under the Health Act. If the Government approves of the proposal, Dr. Norris suggests that the management should be vested in a board of 'flve members, two representing the councils and two representing the Government (such as the Inspector of Charities and the Chairman of the Board of Public Health), with the Minister of Health as Chairman. Dr. Norris's report is, it is added, to be considered by the Cabinet. 